\title{
A New Structural Approach for Mining Frequent Itemset
}

\author{
Khushpinder Singh \\ Computer Science and Engineering \\ Lovely Professional University, India
}

\begin{abstract}
The problem of mining high quality frequent substructures from a large collection of semi-structured data has recently attracted a lot of research. There are various efficient algorithms available for discovering frequent substructures in a large structured data, where both of the patterns and the data are modeled by labeled unordered trees. In this paper, a new structure based algorithm ENHANCE CLIQUE TREE is proposed which creates the unordered structure of itemsets and gives the perceptive representation of the mining results. It eliminates the limitation of FP-tree which always follows the ordered tree structure and its performance also degrades when the dataset is regularly updated. Here a VERIFY TREE ALGORITHM is added in the proposed algorithm which verifies that whether there is a need to restructure the tree or not when the itemsets are inserted or deleted. It improves the performance so that it will reduce unnecessary space and time used for restructure the tree when there is no need for it.
\end{abstract}

\section{Index Terms}

Association rule mining, frequent itemsets, junction tree

\section{INTRODUCTION}

Data mining applications are continuously developing in various industries such as marketing, banking, health care, insurance, manufacturing and medicine which extract more hidden knowledge that enable to increase business efficiency and grow. It brings a lot of profit to businesses, civilization, governments as well as individual. The collective application of association rule techniques emphasizes the knowledge management process and permits marketing personnel to know their customers well to provide improved quality services For example, market basket analysis is used in marketing to provide imminent information on product combinations that were purchased, when these items were bought and in which sequence by various customers of different ages.

The rapid enhancement of computer technology has increasing data collection, storage and manipulations. As the size and complexity of datasets increases, manual data processing on data analysis has replaced with automatic data processing. This automatic data processing is based on various methods such as association, clustering, classification and decision tree. These methods are applied on large datasets resulting as the mining of data. It extracts various hidden predictive patterns or correlations by analysis the collections of observations in number of fields in large relational databases. For example, a grocery store used the data mining technique to analyze local buying patterns. They discovered that when men bought chips on Thursdays and Sunday, they also tended to buy beer. It also showed that these shoppers typically did their weekly grocery shopping on Sunday. On other weekdays, they bought few items. The retailer concluded that they purchased the beer to have it available for the upcoming weekend. The grocery chain could use this data mining concept to increase their sale.

Over the years, discovering frequent itemsets has been a vital research topic in the data mining. There are thousands of algorithms used to mine frequent itemsets. The efficiency of all those algorithms is depends upon the complexity of structure. There have been increasing demands for efficient methods for discovering patterns in large collections of graph and tree structures. The tree structure based algorithms is based on extended prefix-tree structure which store crucial, quantitative information about frequent patterns. Examples of tree structure based algorithms are FP-tree, CAN-tree, CAST-tree etc.

\subsection{Association Rule Mining}

Association rule mining is discovering frequent patterns, associations and correlations among items which are meaningful to the users and can generate strong rules on the basis of these frequent patterns, which helps in decision support system [7]. These patterns will helps to make efficient sales strategies. Let $I=\left\{i_{1}, i_{2}, \ldots, i_{m}\right\}$ be a set, called as items. Let $\mathrm{D}$ a set of transactions and each transaction $\mathrm{T}$ is a set of items such that $\mathrm{T} \subseteq \mathrm{I}$. Let $\mathrm{X}$ is a set of items. A transaction $\mathrm{T}$ is said to contain $\mathrm{X}$ if and only if $\mathrm{X} \subseteq \mathrm{T}$. An association rule is a proposition of the form $\mathrm{X} \Rightarrow \mathrm{Y}$, where $\mathrm{X} \subset \mathrm{I}, \mathrm{Y} \subset \mathrm{I}$, and $\mathrm{X} \cap \mathrm{Y}=\varnothing[1]$.

The rule $X \Rightarrow Y$ is said to have support $s$, if number of times an items existed in the itemsets. Similarly, the rule $X \Rightarrow Y$ is said to have confidence $\mathrm{c}$, if one item have some relation with other item. For example $40 \%$ of transactions that contain bread also contain jam; $7 \%$ of all transactions contain both bread and jam. Here, $40 \%$ is called the confidence $\mathrm{c}$ of the rule, and $7 \%$ the support of the rule. The selection of association rule is based on support and confidence. The confidence factor shows the strength of the inference rules, i.e. the confidence for an association rule is the ratio of the number of transactions that contain XUY to the number of transactions that contain $\mathrm{X}$; whereas the support factor indicates the frequencies of the occurring relations in the rule i.e. the support for an association rule is the percentage of transactions in the database that contain XUY. 


\subsection{Frequent itemsets}

A set of items that appear occur frequently together in a transaction data set, is a frequent itemset. There are various algorithm used to find the frequent itemsets from a dataset. The most common application of frequent itemsets is market basket which analyzes larger sets of items that occur much more frequently. For example, many people buy hot dogs and mustard together. They can advertise a sale on hot dogs and raise the price of mustard. When people come to the store for the low-priced hot dogs, they often will remember that they need mustard, and buy that too.

\section{PRESENT WORK}

\subsection{Problem Formulation}

In 2004, Jaiwei Han has proposed a tree structural approach "Mining Frequent Patterns without Candidate Generation: A Frequent-Pattern Tree Approach". A frequent-pattern tree (FP-tree) is a compact data structure which store critical, quantitative information about frequent patterns. It removes the limitation of Apriori algorithm i.e. there is no candidate generation. It uses three techniques to achieve the efficiency in mining. First, a large database is compressed into a smaller data structure by eliminating the unnecessary repeated database scans. Second, it adopts a patternfragment growth method to avoid the costly generation of a large number of candidate sets. Third, divide-and-conquer method is used to decompose the mining task into a set of smaller tasks for mining confined patterns in conditional databases, which considerably reduces the search space.

The tree structure of FP-tree consists of:-

- Root node, which act as a parent node and all other node are connected to it in a hierarchical structure

- Each node consists of three fields: Itemname, Count, and Node-link. Item-name is the name of the node, count is the number of times it occurs, node link specify the link which points the address of next node.

- $\quad$ Each entry in the header table consists of two fields: Item name and their head of node-link. Here, item-name specifies the name of item and head of node-link specify the link which points to address of next node.

There are some problem definitions in FP-tree approach:

- It always has to follow the order. For example we have items as given
Table 1: Item sets

\begin{tabular}{|c|c|}
\hline TID & ITEMS \\
\hline 100 & $\mathrm{f}, \mathrm{c}, \mathrm{a}, \mathrm{m}, \mathrm{p}$ \\
\hline 200 & $\mathrm{f}, \mathrm{c}, \mathrm{m}, \mathrm{p}, \mathrm{b}$ \\
\hline 300 & $\mathrm{c}, \mathrm{a}, \mathrm{f}, \mathrm{m}, \mathrm{p}$ \\
\hline 400 & $\mathrm{a}, \mathrm{c}, \mathrm{m}, \mathrm{p}, \mathrm{m}$ \\
\hline 500 & $\mathrm{f}, \mathrm{b}$ \\
\hline
\end{tabular}

As we can see that all itemsets are not in order. So if we construct the FP-tree without ordering the itemsets then it will utilize more memory as well as time. To improve the performance of FP-tree, we have to order the itemsets based on their support. If we have a large dataset, then it is utilize lot of space and time in ordering the item sets.

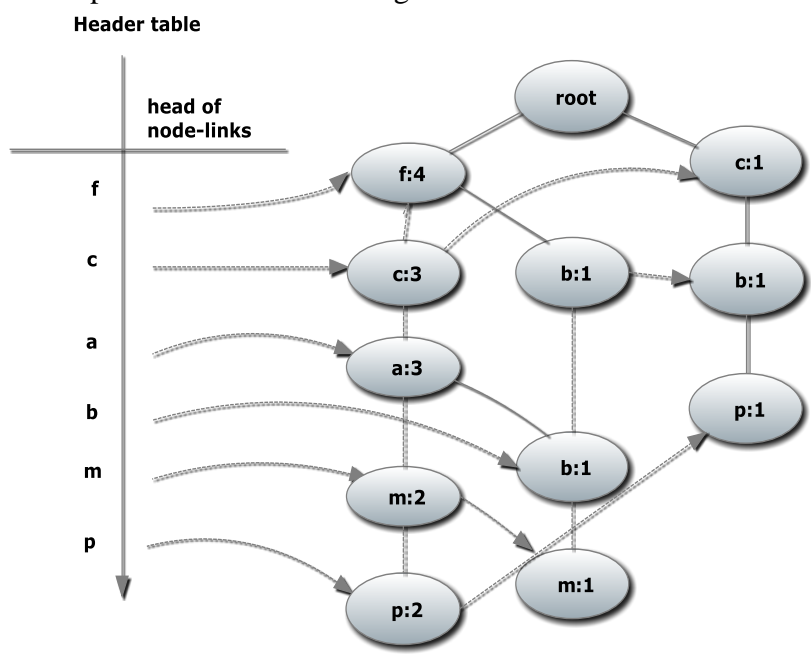

Figure 1: An example of FP-tree

- Another limitation of FP-tree is that it is not efficient in incremental database i.e. when the database is updated very fast. This limitation is related to first one because if the dataset is updated regularly i.e. itemsets are added or removed then again we have to find the support of all the items present in the dataset and reorder it according to their support. This limitation degrades the performance of FP tree.

\subsection{Proposed Work}

We recover the first limitation of FP-tree by introducing a special tree structure, called a junction or clique tree which does not follow any order. It is an unordered structure graph based on the notion of decomposable families of item sets which define a probabilistic model for the data from which the original collection of itemsets was derived. It provides a perceptive representation of the mining results. Junction 
trees are not used as a direct model but rather as a technique for decomposing directed acyclic graph models. This model provides leverage for problems that could be intractable using the entire collection of itemsets. This algorithm is reducing itemset mining results into a smaller and a more manageable group of itemsets.

The second problem formulation is that FP-tree is not efficient when the dataset is updated regularly. It is solved by modified an existing Clique tree algorithm proposed in research paper "Decomposable families of item sets" by Nikolaj Tatti and Hannes Heikinheimo in 2008. This proposed algorithm verifies that whether there is a need to restructure the tree or not when the items are inserted into the dataset.

\subsubsection{Proposed Algorithm}

The proposed ENHANCE CLIQUE TREE algorithm is performed in three steps:

Step1. First of all SEARCH TREE algorithm is performed for computing multiple decomposable covers from a single candidate set $\mathrm{F}$.

Step2. After that MODIFY TREE algorithm is performed which restructure the tree when the new clique is added and also removes the possible cycles.

Step3. After that VERIFIY TREE algorithm is performed which verifies that whether there is a need to restructure the tree or not

ALGORITHM 1: SEARCH TREE ALGORITHM. The input is a downward closed cover $\mathrm{F}$, the output is a junction tree $\mathrm{T}$ such that $\mathrm{V}(\mathrm{T}) \subseteq \mathrm{F}$.

1. Set $\mathrm{V}(\mathrm{T} 0) \leftarrow\{\mathrm{x} ; \mathrm{x} \in \mathrm{A}\}$

2. Set $\mathrm{n} \leftarrow 0$

3. Repeat

$$
\text { 4. } n \leftarrow n+1
$$$$
\text { 5. } \mathrm{T}_{\mathrm{n}} \leftarrow \mathrm{T}_{\mathrm{n}-1}
$$$$
\text { 6. } \mathrm{V}(\mathrm{Gn}) \leftarrow\{\mathrm{X} \in \mathrm{V}(\mathrm{Tn}) ;|\mathrm{X}|=\mathrm{n}\}
$$

7. $\mathrm{E}(\mathrm{Gn}) \leftarrow\{(\mathrm{X}, \mathrm{Y}) ; \mathrm{X}, \mathrm{Y} \in \mathrm{V}(\mathrm{Gn}), \mathrm{X} \cap \mathrm{Y} \mid=\mathrm{n}-1, \mathrm{X} \cup \mathrm{Y} \in \mathrm{F}\}$

8. Calculate $\mathrm{w}(\mathrm{x})$ for each $(\mathrm{X}, \mathrm{Y})$ and store as

Edge []$=(X, Y)$

Weight []$=\mathrm{w}(\mathrm{x})$

Flag []$=0$

9. Repeat

e=edge []$\leftarrow \arg \max x \in E($ gn $) w(x)$

Flag $[\mathrm{e}]=1$;

If $\mathrm{X}$ and $\mathrm{Y}$ are $\mathrm{n}-1$ connected in $\mathrm{Tn}$ then call MODIFY algorithm

End if

Until $\mathrm{E}(\mathrm{Gn})=$ NULL

Delete nodes marked by MODIFY TREE from Tn connected the incident nodes.

Until Gn is empty

Return T.

$$
\begin{aligned}
& \text { ALGORITHM 2: MODIFY TREE ALGORITHM } \\
& \text { 1. } \mathrm{B} \leftarrow \mathrm{X} \cup \mathrm{Y} \\
& \text { 2. } \mathrm{V}(\mathrm{Tn}) \leftarrow \mathrm{V}(\mathrm{Tn}) \mathrm{U}\{\mathrm{B}\} \text {. }\{\text { Add new clique into } \mathrm{Tn}\} \\
& \text { 3. For } \mathrm{v} \in \mathrm{B} \text { do } \\
& \mathrm{W} \leftarrow \mathrm{B}-\mathrm{v} \\
& \mathrm{MARK} \text { W } \\
& \text { If } \mathrm{W} \notin \mathrm{V}(\mathrm{Gn}) \text { then } \\
& \quad \mathrm{V}(\mathrm{Gn}) \leftarrow \mathrm{V}(\mathrm{Gn}) \cup\{\mathrm{W}\} \\
& \mathrm{E}(\mathrm{Gn}) \leftarrow \mathrm{E}(\mathrm{Gn}) \mathrm{U}\{\mathrm{W}, \mathrm{Z} ; \mathrm{Z} \in \mathrm{V}(\mathrm{Gn}),|\mathrm{X} \cap \mathrm{Z}|=\mathrm{n}-1, \mathrm{~V} \\
& \neq \mathrm{X} \cup Z \in \mathrm{F}\} \quad \mathrm{V}[\mathrm{Tn}] \leftarrow \mathrm{V}(\mathrm{Tn}) \cup\{\mathrm{W}\} \\
& \text { end if }
\end{aligned}
$$

end for

$$
\mathrm{E}(\mathrm{Tn}) \leftarrow \mathrm{E}(\mathrm{Tn}) \mathrm{U}(\mathrm{B}, \mathrm{W}) .
$$

Remove the possible cycle in Tn by removing an edge (U, $\mathrm{V})$ connecting $\mathrm{X}$ and

$\mathrm{Y}$ and having $|\mathrm{U} \cap \mathrm{V}|=\mathrm{n}-1$.

\section{ALGORITHM 3: VERIFY TREE ALGORITHM}

1. For each $\mathrm{A} \in \mathrm{V}\{\mathrm{Tn}\}$

2. For each a€A weight \{\}

Calculate w(a) and sort them S_Edge[ ] [ ]

Flag 2 $=0$;

For $\mathrm{i}=0$ to $|\mathrm{V}\{\mathrm{T} 0\}|$

$$
\{
$$$$
\text { If(edge [ i ] [ } 2 \text { ]!= S_Edge [ i ] [2]) }
$$

\{

Set flag 2=1;

$$
\text { \} }
$$

Break;

\section{\}}

End for loop.

If (flag2==1)

$$
\text { Then call SEARCH TREE ALGORITHM }
$$

Else tree remains unchanged.

\subsubsection{Working Of Enhance Clique Tree Algorithm}

The proposed algorithm is explained briefly as:

First of all, SEARCH TREE algorithm is performed which finds the tree with low entropy. It is used for computing multiple decomposable covers from a single candidate set F. The aim is to find a decomposable downward closed family covering the set of attributes. At the beginning of each iteration round we have a junction tree $\mathrm{T}$ whose cliques have at most $\mathrm{n}$ attributes. The first tree $\mathrm{T} 0$ is created which containing only single attributes and no edges. During each round, the tree is modified so that in the end we will have Tn, a tree with cliques having at most $n+1$ attributes. Here, $\mathrm{X}$ and $\mathrm{Y}$ are said to be $\mathrm{n}-1$ connected in a junction tree $\mathrm{T}$, if there is a path in $\mathrm{T}$ from $\mathrm{X}$ to $\mathrm{Y}$ having at least one separator of size $\mathrm{n}-1$. We say that $\mathrm{X}$ and $\mathrm{Y}$ are 0 -connected, if $\mathrm{X}$ and $\mathrm{Y}$ are not connected.

Each round of the algorithm is consists of three steps:

- Generate: A graph is constructed and add all the edges to the graph. A weight is assign to each edge such that $\mathrm{w}(\mathrm{e})=\mathrm{H}(\mathrm{X})+\mathrm{H}(\mathrm{Y})-\mathrm{H}(\mathrm{X} \cap \mathrm{Y})-\mathrm{H}(\mathrm{X} \cup \mathrm{Y})$

Augment: We select the edge e which have the largest weight and remove it from graph. If $\mathrm{X}$ and $\mathrm{Y}$ are $\mathrm{n}-1$ connected in $\mathrm{Tn}$ tree we add $\mathrm{Tn}$ with a new clique $\mathrm{V}=\mathrm{X} \cup \mathrm{Y}$.

- $\quad$ Purge: The tree $T_{n}$ contains redundant cliques after augmentation. We remove these redundant cliques from $T_{n}$.

After that MODIFY TREE algorithm removes the possible cycles in $\mathrm{T}_{\mathrm{n}}$ by removing an edge $(\mathrm{U}, \mathrm{V})$ connecting $\mathrm{X}$ and $\mathrm{Y}$ and having $|\mathrm{U} \cap \mathrm{V}|=\mathrm{n}-1$

At last, VERIFY TREE algorithm is performed which verifies that whether there is need for recreation of the tree or not. It will improve the performance so that it will avoid unnecessary space and time. It verifies it based on the weights assign to each edge.

$$
\mathrm{w}(\mathrm{e})=\mathrm{H}(\mathrm{X})+\mathrm{H}(\mathrm{Y})-\mathrm{H}(\mathrm{X} \cap \mathrm{Y})-\mathrm{H}(\mathrm{X} \cup \mathrm{Y})
$$

where $\mathrm{H}(\mathrm{X})$ and $\mathrm{H}(\mathrm{Y})$ is the entropy of $\mathrm{X}$ and $\mathrm{Y}$ respectively.

We have stored the value of weight $w(x)$ of each $(X, Y)$ in the array.

When the items need to be inserted, first check its (n-1) connected. When the count increased, first check the weights with its (n-1) connected. If the weight is higher than the 
previous one then the tree need to be restructure otherwise no change in the representation.

\section{RESULTS}

In this section, a performance comparison of two association rule mining algorithms i.e. FP-growth tree and proposed clique growth tree algorithm is presented.

The two association rule mining algorithms were tested in WEKA software of version 3.7.9. WEKA software is a collection of open source of many data mining and machine learning algorithms, including pre-processing on data, Classification, clustering and association rule extraction [8]. It had tested on window XP operating system. The performance of FP-growth and clique tree was evaluated based on execution time. The execution time is the total time taken to generate the rules.

We compare the performance of these two algorithms as follow:

- We generate the 5 different datasets i.e. 5000, $10000,15000,20000,25000$

- Then we apply these two algorithms on these 5 dataset in WEKA

- Saves the results in the excel

- Plot a graph which compares the results in terms of total execution time.

Table 2: Results shown in the table form

\begin{tabular}{|c|c|c|}
\hline Datasets & $\begin{array}{l}\text { FP-Growth tree } \\
\text { (execution time in } \\
\text { ns) }\end{array}$ & $\begin{array}{l}\text { Clique tree } \\
\text { growth(execution } \\
\text { time in ns) }\end{array}$ \\
\hline 5000 & 219 & 172 \\
\hline 10000 & 297 & 234 \\
\hline 15000 & 500 & 313 \\
\hline 20000 & 578 & 406 \\
\hline 25000 & 719 & 532 \\
\hline
\end{tabular}

In table 2, results are shown in the form of tables. First column shows the number of dataset used, second column shows the time taken by FP-growth tree algorithm and third column shows the time taken by clique tree growth algorithm. The results that the clique tree growth algorithm always take less time as compare to FP-growth tree algorithm.

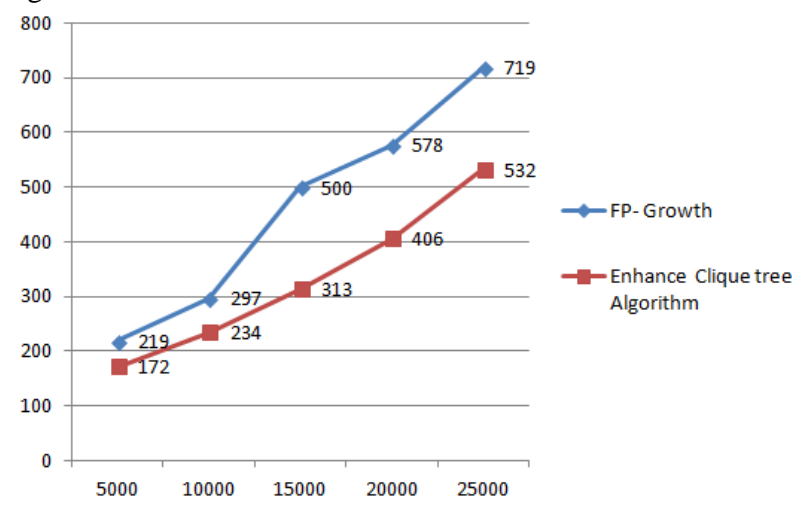

Figure 2: Plot a line graph based on results
It shows the comparisons of two algorithms in terms of total execution time. Based on these comparisons, it can say that the performance of FP-growth tree degrades as the dataset increases. But on the other hand, clique tree shows the consistent results on the basis of time.

\section{CONCLUSION}

Association rules have been broadly used in many applications domains for effective decision making. It seeks to discover associations among transactions encoded in a database. Market basket analysis is the broad application used to discover the pattern reveals combinations of events that occur at the same time. In this paper, we present an efficient algorithm to build decomposable itemset families which improve the limitations of FP-tree. We introduced a VERIFY TREE algorithm which verifies that whether there is a need to restructure the tree or not when the dataset is updated. It shows the empirical relationship between the decomposable itemset families and the candidate set and the performance of Boolean queries using multiple decomposable families. It provides high quality results in incremental database due to the expressiveness and good interpretability of the model. Our results show that the performance of FP-growth tree degrades as the dataset increases where as clique tree shows the consistent results on the basis of time.

\section{ACKNOWLEDGMENT}

I would like to thank my mentor for being a great motivator and helping me in all aspects, also I will thank my friends to motivate me for writing the paper.

\section{REFERENCES}

[1] Rakesh Aggarwal, Ramakrishnan Srikant, (1994) "Fast algorithm for mining association rule" Proceeding on 20th VLDB Conference Santiago, Chile.

[2] Nikolaj Tatti, Hannes Heikinheimo, (2008) "Decomposable families of Itemsets" Helsinki University of Technology, Finland.

[3] Arabinda Nanda, Saroj Kumar Rout, (2008) "Data Mining \& Knowledge Discovery in Databases: An AI Perspective" Department of Computer Science, Gandhi Engineering college, Bhubaneswar.

[4] Jiawei Han jain pei, Yiwen Yin, Runying mao, (2004) "Mining Frequent Patterns without Candidate Generation: A Frequent-Pattern Tree Approach" Data Mining and Knowledge Discovery, 8, 53-87

[5] Sanjeev Rao, Priyanka Gupta, (2012) "Implementing Improved Algorithm Over APRIORI Data Mining Association Rule Algorithm" IJCST Vol 1.3, Jan-March

[6] Akash Rajak, Mahendra Kumar Gupta, (2007) "Association Rule Mining: Applications in Various Areas" Krishna Institute of Engineering \& Technology, Ghaziabad

[7] http://www.coolinterview.com/interview/36017

[8] 8)http://in.docsity.com/endocs/Introduction_to_WEKA _Part_1-Data_Warehouse-Lecture_Handout_ 